Shtegman V.O., Morozov A.V., Freidin A.B., Müller W.H. On stress-affected kinetics of intermetallic compound growth in the presence of electromigration // Вестник Пермского национального исследовательского политехнического университета. Механика. 2020. - № 4. - C. 7-14. DOI: 10.15593/perm.mech/2020.4.01

Shtegman V.O., Morozov A.V., Freidin A.B., Müller W.H. On stress-affected kinetics of intermetallic compound growth in the presence of electromigration. PNRPU Mechanics Bulletin, 2020, no. 4, pp. 7-14. DOI: 10.15593/perm.mech/2020.4.01

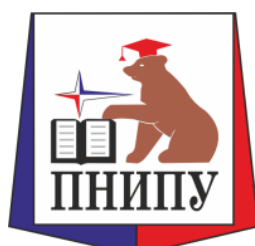

ВЕСТНИК ПНИПУ. МЕХАНИКА

№ 4, 2020

PNRPU MECHANICS BULLETIN

https://ered.pstu.ru/index.php/mechanics/index

DOI: $10.15593 /$ perm.mech/2020.4.01

UDC 539.3

\title{
ON STRESS-AFFECTED KINETICS OF INTERMETALLIC COMPOUND GROWTH IN THE PRESENCE OF ELECTROMIGRATION
}

\author{
V.O. Shtegman ${ }^{1,2}$, A.V. Morozov ${ }^{2,3}$, A.B. Freidin ${ }^{1,2,4}$, W.H. Müller ${ }^{3}$ \\ ${ }^{1}$ Saint Petersburg University, St. Petersburg, Russian Federation \\ ${ }^{2}$ Institute for Problems in Mechanical Engineering of the Russian Academy of Sciences, \\ St. Petersburg, Russian Federation \\ ${ }^{3}$ Technische Universität Berlin, Berlin, Germany \\ ${ }^{4}$ Peter the Great St. Petersburg Polytechnic University, St. Petersburg, Russian Federation
}

\section{ARTICLE INFO}

Received: 09 November 2020

Accepted: 15 December 2020

Published: 30 December 2020

\section{Keywords:}

mechanochemistry, intermetallic compounds, lead-free solder, chemical affinity tensor, chemical reaction kinetics, diffusion, electromigration, internal stresses, vacancies,

Kirkendall voids.

\begin{abstract}
This paper is concerned with the analytical modeling of an intermetallic compound formation in a eutectic tin solder joint on copper interconnects subjected to an electrical current. We propose a model that couples mechanical stresses, chemical reaction, diffusion, temperature, and electromigration. The kinetics of the chemical reaction fronts of the intermetallic phase formation is investigated based on the notion of the chemical affinity tensor within the small strain approximation. It allows incorporating the influence of stresses and strains on the chemical reaction rate and the normal component of the reaction front velocity in a rational manner. Electromigration is introduced into the model as an additional summand in the total flux of the diffusive constituents, which, in turn, also affects the reaction front velocity. In the considered model, the mechanical stresses arise due to the internal strains produced by the chemical transformation and by the thermal expansion. We formulate a model problem for planar reaction fronts. Within this model, the influence of stresses and electromigration on the reaction front kinetics is studied analytically. Based on the Mean-Time-To-Failure (MTTF) criteria, we calculate the critical thickness of the solder joint and estimate the amount of the accumulated vacancies. We introduce a dimensionless parameter, which characterizes the accumulation of vacancies due to electromigration enhanced diffusion. Finally, we discuss the coupling between the accumulated vacancies and Kirkendall void nucleation.
\end{abstract}




\section{Introduction}

In modern microelectronic devices lead-free solders are used for creating electrical and mechanical contact between microelectronic circuit components. During the last few decades technological progress relied heavily on the miniaturization of electronic devices making it crucial to account for the micro- and nanosized effects in solders. One of the most common $\mathrm{Pb}$-free solder is $\mathrm{Sn} 3.0 \mathrm{Ag} 0.5 \mathrm{Cu}$ [1]. After soldering an Inter-Metallic Compound (IMC) layer forms and establishes a mechanical contact between the tin-based solder bump and the copper interconnect of the microelectronic circuit, Fig. 1. During further use of the microelectronic component, the IMC layer grows considerably, especially when the component is exposed to heat [2] and strong electric current [3]. Intermetallics are brittle in comparison to pure $\mathrm{Cu}$ or $\mathrm{Sn}$ materials. Also, IMC formation is based on multi-component diffusion which may lead to fracture due to Kirkendall voiding [4]. In addition, void formation may be enhanced by electromigration [5]. Thus the reliability of the solder joint highly depends on the thickness and the void formation in the IMC layer.

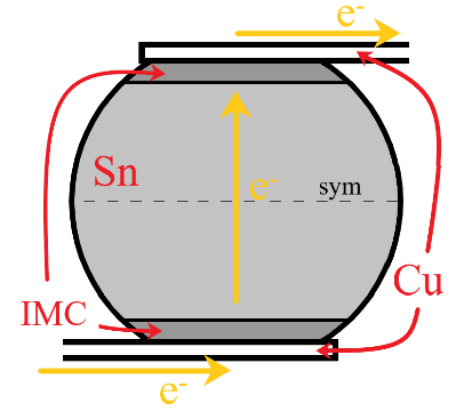

Fig. 1. Schematic representation of a solder joint cross-section.

This paper is organized as follows. In Section 2 we give a concise overview of the IMC phase growth and subsequent Kirkendall void nucleation. In Section 3 an analytical approach to the coupled problem of diffusion, mechanics, and chemical reaction is presented. The growth kinetics of the IMC is investigated based on the notion of the chemical affinity tensor within the small strain approximation. The influence of mechanical stresses is accounted for through their influence on the chemical affinity tensor on which the reaction front velocity depends. Electromigration is considered as an additional summand in the total flux of the diffusive constituents. In Section 4 the kinetics of the IMC growth under electrical current stressing in an infinite layer is analyzed. A brief overview of void nucleation is given in Section 5. Finally, conclusions and an outlook are provided in Section 6.

\section{Problem Formulation}

As a result of melting and subsequent solidification of a solder material an IMC layer forms between the $\mathrm{Cu}$ substrate and the Sn-based solder. At the operating temperatures of modern microelectronic devices with $\mathrm{Sn} 3.0 \mathrm{Ag} 0.5 \mathrm{Cu}$ solder the formation of two intermetallic phases becomes possible: $\mathrm{Cu}_{3} \mathrm{Sn}$, also known as the $\varepsilon$-phase, and $\mathrm{Cu}_{6} \mathrm{Sn}_{5}$, also known as the $\eta^{\prime}$-phase. The growth kinetics of the $\eta^{\prime}$ phase was identified as predominant by several experimental and theoretical studies [6-8]. Thus, in what follows only the growth of the $\eta^{\prime}$ phase is considered. A schematic representation of the solder interconnect is shown in Fig. 1. The formation of the $\eta^{\prime}$ intermetallic phase proceeds according to the following chemical reaction between $\mathrm{Cu}$ and $\mathrm{Sn}$ :

$$
6 \mathrm{Cu}+5 \mathrm{Sn} \rightarrow \mathrm{Cu}_{6} \mathrm{Sn}_{5} .
$$

During use the microelectronic component is subjected to high current stressing of approximately $10^{3}-10^{4} \mathrm{~A} / \mathrm{cm}^{2}$ under typical operating temperatures of $22^{\circ} \mathrm{C}-100^{\circ} \mathrm{C}$, as a result of which the IMC layer grows considerably. During that process $\mathrm{Cu}$ atoms diffuse through the $\eta^{\prime}$ phase to the $\eta^{\prime}-$ $\mathrm{Sn}$ interface, where the chemical reaction (1) is taking place. Simultaneously $\mathrm{Sn}$ atoms diffuse through the $\eta^{\prime}$ phase to the $\mathrm{Cu}-\eta^{\prime}$ interface, where the chemical reaction is taking place as well. The diffusion in binary $\mathrm{Cu}-\mathrm{Sn}$ occurs by a vacancy mechanism [9]. Hence, there is a vacancy flux of opposite sign and equal in magnitude to the total flux of $\mathrm{Cu}$ and of Sn. Differences of these fluxes result in the accumulation of vacancies and, therefore, in the formation of Kirkendall voids [6]. The diffusivity of $\mathrm{Cu}$ is greater than of $\mathrm{Sn}$. Hence, vacancies tend to accumulate near the $\eta^{\prime}-\mathrm{Cu}$ interface, see, e.g., [2]. These voids have been identified as a primary cause of solder joint failure [10-12].

Multiple factors may affect the amount of the accumulated vacancies: (1) Mismatch of the diffusivities of the species; (2) Temperature; (3) Mechanical stresses; (4) High current density in the solder interconnect. In general, all of these factors are coupled. Mismatch of the diffusivities of the materials results in vacancy diffusion and accumulation near the IMC-Cu boundary due to the Kirkendall effect. The diffusivities of $\mathrm{Cu}$ and $\mathrm{Sn}$ depend on the temperature, e.g., $D_{T=20^{\circ} \mathrm{C}}^{\mathrm{Cu}}=4.32 \cdot 10^{-16}\left[\mathrm{~cm}^{2} / \mathrm{s}\right][13]$ and $D_{T=150^{\circ} \mathrm{C}}^{\mathrm{Cu}}=7.04 \cdot 10^{-12}\left[\mathrm{~cm}^{2} / \mathrm{s}\right]$ [7]. The high current density results in mass transfer along the direction of the current due to the presence of the electron wind force (momentum exchange between the moving electrons and ionic atoms due to scattering of valence electrons), which in turn affects the diffusion. Depending on the direction of the electric current it can expedite or retard the diffusion process. Strictly speaking, the current distribution is not uniform in the solder interconnect. This results in a current crowding effect and socalled "back stresses" [14]. Additionally, the strong electric current results in a nonuniform temperature distribution in the solder ball (Joule heating effect). The temperature gradient is the source of thermomigration. At operating temperatures (up to $150{ }^{\circ} \mathrm{C}$ ) thermal strains in combination with the transformation strains (as the result of the chemical reaction) result in mechanical stresses. These stresses may influence the reaction rate and the diffusion coefficient. 


\section{Modeling}

During modeling of the reaction front kinetics, the notion of the chemical affinity tensor is used. It was obtained as a direct result from the mass balance, momentum balance, energy balance, and the second law of thermodynamics in terms of the Clausius-Duhem inequality for moving reaction front in [15]. The normal component of a chemical affinity tensor acts as configurational force on which the reaction front velocity depends. Effects of stresses and strains on the reaction front kinetics are accounted for by their influence on the chemical affinity tensor. Detailed derivations of the chemical affinity tensor are presented in [16]. The validity of the chemical affinity tensor approach for the modeling of the reaction front kinetics was demonstrated in bodies of various rheology [16, 17, 19-21].

Furthermore, the chemical reaction (1) can be generalized as:

$$
n_{-} B_{-}+n_{*} B_{*} \rightarrow n_{+} B_{+},
$$

where $n_{-}, n_{*}$, and $n_{+}$are the stoichiometric coefficients, $B_{-}, B_{*}$, and $B_{+}$are the chemical formulas of the constituents in the reaction, and the subscripts "-", “*”, and “+” refer to the initial material, diffusive reactant, and reaction product, respectively. It is assumed that the chemical reaction is localized at the reaction front $\Gamma$ and that all of the diffusive reactant is consumed during the reaction. The chemical reaction is accompanied by a change in volume, which one can characterize as [20]:

$$
J_{\mathrm{ch}}=\frac{n_{+} M_{+} / \rho_{+}+\xi n_{*} M_{*} / \rho_{*}}{n_{-} M_{-} / \rho_{-}},
$$

where $M_{-}, M_{+}$, and $M_{*}$ are the molar masses, $\rho_{-}, \rho_{+}$, and $\rho_{*}$ are the densities of the constituents, and $\xi$ is a fitting parameter. The case $\xi=0$ corresponds to the solid skeleton approach (diffusion is not accompanied by the change of volume) and the case $\xi=1$ corresponds to adding the volumes of the reaction product and of the diffusive reactant.

The kinetic equation used by Glansdorff and Prigogine [22] can be reformulated for the reaction rate $\omega$ at the area element with the normal $\mathbf{n}$ as follows [23]:

$$
\omega_{n}=k_{*} c\left\{1-\exp \left(-\frac{A_{n n}}{R T}\right)\right\},
$$

where $k_{*}$ is a kinetic coefficient, $c$ is the partial molar concentration of the diffusive reactant, $R$ is the universal gas constant, $T$ is the current temperature, and $A_{n n}$ is the normal component of chemical affinity tensor.

The normal component of the reaction front velocity, $W_{n}$, can be obtained from the mass balance at the reaction front:

$$
W_{n}=\frac{n_{-} M_{-}}{\rho_{-}} \omega_{n} .
$$

A linear-elastic material response assumption is used. The constitutive equations read:

$$
\boldsymbol{\sigma}_{-}=\mathbf{C}_{-}:\left(\varepsilon_{-}-\varepsilon_{-}^{\text {th }}\right), \sigma_{+}=C_{+}:\left(\varepsilon_{+}-\varepsilon_{-}^{\text {th }}-\varepsilon^{\text {ch }}\right),
$$

where $\mathbf{C}_{ \pm}$are stiffness tensors, $\boldsymbol{\varepsilon}_{ \pm}^{\text {th }}$ and $\boldsymbol{\varepsilon}^{\text {ch }}$ are the thermal and the chemical transformation strains, which are given by:

$$
\begin{gathered}
\mathbf{C}_{ \pm}=\lambda_{ \pm} \mathbf{E} \otimes \mathbf{E}+2 \mu_{ \pm}{ }^{4} \mathbf{I}, \\
\boldsymbol{\varepsilon}_{ \pm}^{\text {th }}=a_{ \pm}\left(T-T_{0}\right) \mathbf{E}, \boldsymbol{\varepsilon}^{\mathrm{ch}}=\varepsilon^{\mathrm{ch}} \mathbf{E}=\left(J_{\mathrm{ch}}^{1 / 3}-1\right) \mathbf{E},
\end{gathered}
$$

where $\lambda_{ \pm}$and $\mu_{ \pm}$are the Lamé parameters, $a_{ \pm}$are the coefficients of thermal expansion, $T_{0}$ is the reference temperature, $\mathbf{E}$ and ${ }^{4} \mathbf{I}$ are the second and forth order unit tensors, respectively. During further analysis the stoichiometric coefficients $n_{*}, n_{-}, n_{+}$are normalized by $n_{*}\left(n_{-} \rightarrow n_{-} / n_{*}\right.$, $\left.n_{+} \rightarrow n_{+} / n_{*}\right)$.

It could be shown [16] that the expression for the normal component of the chemical affinity tensor in accordance with quasi-statics and a small strains approximation has the following form:

$$
\begin{gathered}
A_{n n}=\frac{n_{-} M_{-}}{\rho_{-}}\left\{\gamma(T)+\frac{1}{2} \boldsymbol{\sigma}_{-}:\left(\boldsymbol{\varepsilon}_{-}-\boldsymbol{\varepsilon}_{-}^{\mathrm{th}}\right)-\right. \\
\left.-\frac{1}{2} \boldsymbol{\sigma}_{+}:\left(\boldsymbol{\varepsilon}_{+}-\boldsymbol{\varepsilon}_{+}^{\mathrm{th}}-\boldsymbol{\varepsilon}^{\mathrm{ch}}\right)+\boldsymbol{\sigma}_{-}:\left(\boldsymbol{\varepsilon}_{+}-\boldsymbol{\varepsilon}_{-}\right)\right\}+R T \ln \left(\frac{c}{c_{*}}\right),
\end{gathered}
$$

where $\gamma(T)$ is the chemical energy parameter and $c_{*}$ is the reference concentration of the diffusive constituent.

In order to determine the kinetics of the reaction front one has to obtain the concentration, $c$, of the diffusing reactant and the mechanical stresses, $\boldsymbol{\sigma}$, and strains, $\boldsymbol{\varepsilon}$, at the interface. To do so, a steady-state diffusion problem with stationary diffusion equation and a quasi-static elasticity problem have to be solved. Then, the obtained values are used to compute the normal component of the chemical affinity tensor (8) and the reaction rate (4), which defines the reaction front velocity according to (5).

Each solder interconnect has two IMC layers (Fig. 1) and they are assumed to be symmetrical about the middle plane of the solder. In order to investigate the effects of electromigration on the growth kinetics of each IMC layer one has to consider two cases (Fig. 2).

According to experimental data [18] the solder ball diameter greatly exceeds the thickness of the IMC layer. This gives reason to formulate a boundary value problem for an infinite layer with a plane reaction front (Fig. 2).

The mechanical equilibrium equation,

$$
\nabla \cdot \boldsymbol{\sigma}=0,
$$

with boundary conditions,

$$
\begin{gathered}
u_{y}^{\mathrm{Cu}}(0)=0, u_{y}^{\mathrm{Cu}}(a)-u_{y}^{\mathrm{n}^{\prime}}(a)=0, \\
u_{y}^{\mathrm{Sn}}(b)-u_{y}^{\eta^{\prime}}(b)=0,
\end{gathered}
$$



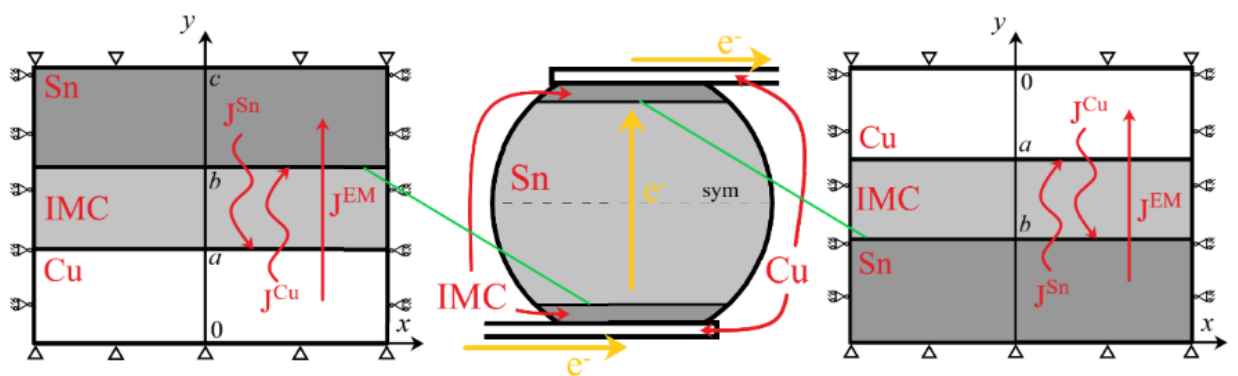

Fig. 2. The two considered cases of the model problem

$$
\begin{gathered}
\sigma_{y y}^{\mathrm{Cu}}(a)-\sigma_{y y}^{\mathrm{n}^{\prime}}(a)=0, \quad \sigma_{y y}^{\mathrm{Sn}}(b)-\sigma_{y y}^{\mathrm{n}^{\prime}}(b)=0, \\
u_{y}^{\mathrm{Sn}}(c)=0,
\end{gathered}
$$

can be satisfied by the following displacement fields:

$$
\mathbf{u}^{k}(y)=u_{y}^{k}(y) \mathbf{e}_{y}=\left(A^{k} y+B^{k}\right) \mathbf{e}_{y},
$$

where $k$ corresponds to $\mathrm{Cu}, \mathrm{Sn}$, or $\eta^{\prime}$. The constants $A^{k}$ and $B^{k}$ can be found from the boundary conditions (10) and (11).

Each of the considered cases consists of two reactions happening simultaneously at different reaction fronts. Hence, four stationary diffusion problems are considered with the assumption that the fluxes of $\mathrm{Cu}$ and of $\mathrm{Sn}$ do not interfere. For the sake of clarity, the four diffusion problems are further referred as: (1) Reaction at the $\eta^{\prime}-\mathrm{Cu}$ interface, $J^{\mathrm{EM}}$ is directed opposite to $J^{\mathrm{D}}$ (Fig. 2a); (2) Reaction at the $\eta^{\prime}$-Sn interface, $J^{\mathrm{EM}}$ is co-directed with $J^{\mathrm{D}}$ (Fig. 2a); (3) Reaction at the $\eta^{\prime}-\mathrm{Cu}$ interface, $J^{\mathrm{EM}}$ is co-directed with $J^{\mathrm{D}}$ (Fig. 2b); (4) Reaction at the $\eta^{\prime}-\mathrm{Sn}$ interface, $J^{\mathrm{EM}}$ is directed opposite to $J^{\mathrm{D}}$ (Fig. 2b); where $J^{\mathrm{D}}$ is the Fickian flux, and $J^{\mathrm{EM}}$ is the flux due to electromigration. Later all indexes on the material parameters are omitted, because all material parameters correspond to the diffusive material in each considered case, unless otherwise is stated.

Therefore, the total fluxes for the considered cases have the form:

$$
\begin{aligned}
\text { 1) } J^{\Sigma}=-J^{\mathrm{D}}+J^{\mathrm{EM}}, \text { 2) } J^{\Sigma}=J^{\mathrm{D}}+J^{\mathrm{EM}}, \\
\text { 3) } J^{\Sigma}=-J^{\mathrm{D}}-J^{\mathrm{EM}}, \text { 4) } J^{\Sigma}=J^{\mathrm{D}}-J^{\mathrm{EM}},
\end{aligned}
$$

where the fluxes are given by (see, e.g., [24, 25]):

$$
J^{\mathrm{D}}=-D \frac{\partial c}{\partial y}, J^{\mathrm{EM}}=\frac{D}{L} c, L=\frac{k T}{Z^{*} e \varrho j},
$$

and $D$ is the diffusivity of the species through the reaction product, $Z^{*}$ is the effective charge number of electromigrations, $k$ is the Boltzmann constant, $e$ is the electron charge, $\varrho$ is the electrical resistivity, and $j$ is the current density.

Consider the equation governing stationary diffusion in the following form,

$$
\frac{\partial J^{\Sigma}}{\partial y}=0
$$

with boundary conditions,

$$
\begin{gathered}
D \mathbf{n} \cdot\left(\nabla c_{i} \pm \frac{Z^{*} e \varrho \mathbf{j}}{k T} c_{i}\right)-a_{*}\left(c_{*}-c_{i}(b)\right)=0, \text { at } \Omega_{+}, \\
D \mathbf{n} \cdot\left(\nabla c_{i} \pm \frac{Z^{*} e \varrho \mathbf{j}}{k T} c_{i}\right)+k_{*}\left(c_{i}(a)-\varkappa c_{*}\right)=0, \text { at } \Gamma,
\end{gathered}
$$

where the index $i$ refers to the considered case introduced above in (13), $a_{*}$ is the surface mass transfer coefficient, $\Omega_{+}$is the boundary between reaction product and diffusive constituent, $\mathbf{n}$ is the normal vector directed outward the reaction product, the input of electromigration is positive in the cases 2 and 3 , and the parameter $\varkappa$ is defined as:

$$
\varkappa=\exp \left(-\frac{n_{-} M_{-}}{\rho_{-}} \frac{\chi}{R T}\right),
$$

where $\chi$ is the term in braces in the expression (8) for $A_{n n}$, representing the influence of the mechanical stresses on the reaction rate. In addition, effects of mechanical stresses can be accounted for through the stress-dependent diffusivity and the cross effects of stress gradient in the diffusion flux, see, e.g., [26]. In the present work we focus on the influence of stresses on the reaction rate.

The first boundary condition in (16) states that when the saturation $c_{*}$ is reached then the supply of the diffusive reactant will stop. The second boundary condition results from the mass balance at the reaction front and states that all of the diffusive reactant is consumed by the reaction.

The solution of the stationary diffusion equation (15)for each formulated problem is:

$$
c_{i}(y)=\beta_{i 1} L_{i}+\beta_{i 2} \exp \left( \pm \frac{y}{L_{i}}\right),
$$

where the sign in the exponent is "+" in cases 1 and 4 , and "-" otherwise. The constants $\beta_{i 1}$ and $\beta_{i 2}$ are obtained for each considered case from the boundary conditions (16).

Once the mechanical and diffusion boundary value problems are solved one can obtain the normal component of the reaction front velocity $W_{n}(h)$ as a function of the interface position. This gives the dependence of the reaction front position on time, i.e., the kinetics of the interface.

In order to investigate solder interconnect electromigration reliability one can determine Mean-TimeTo-Failure (MTTF) according to Black's equation [27]: 


$$
\mathrm{MTTF}=A j^{-n} \exp \left(\frac{Q}{k T}\right),
$$

where $A$ is the experimentally obtained fitting parameter, $n$ is a model parameter for the current density, which depends on the mode of failure, and $Q$ is the activation energy in $\mathrm{eV}$.

Once the reaction front velocity (5) and the moment of failure (19) are determined, one can find a critical position of the reaction front, $h_{\mathrm{cr}}$, for each considered case from:

$$
t_{\mathrm{cr}}(h)=\int_{h_{\mathrm{m}}}^{h_{\mathrm{cr}}} \frac{1}{W_{n}(h)} \mathrm{d} h=\mathrm{MTTF},
$$

where $h_{\text {in }}$ is the initial position of the reaction front.

Since diffusion in binary $\mathrm{Sn}-\mathrm{Cu}$ alloys occurs by a vacancy mechanism one can introduce the amount of vacancies accumulated during the chemical reaction. The diffusion coefficient of $\mathrm{Cu}$ is greater than the one of Sn (Table 1), so the net movement of the vacancies will be from the $\eta^{\prime}-\mathrm{Sn}$ interface to the $\eta^{\prime}-\mathrm{Cu}$ interface. Since the total vacancy flux is equal to the difference between total fluxes of $\mathrm{Cu}$ and $\mathrm{Sn}$, the total amount of vacancies accumulated at the $\eta '-\mathrm{Cu}$ interface can be calculated as:

$$
\begin{gathered}
\Phi^{\mathrm{vac}}=\Phi^{\mathrm{Cu}}-\Phi^{\mathrm{Sn}}, \\
\Phi^{\mathrm{Cu}}=\int_{b_{\mathrm{in}}}^{b_{\mathrm{ve}}} \frac{J_{\mathrm{S}}^{\mathrm{Cu}}}{W_{n}^{\mathrm{Cu}}(b)} \mathrm{d} b, \Phi^{\mathrm{Sn}}=\int_{a_{\mathrm{in}}}^{a_{\mathrm{vf}}} \frac{J_{\mathrm{S}}^{\mathrm{Sn}}}{W_{n}^{\mathrm{Sn}}(a)} \mathrm{d} a .
\end{gathered}
$$

One can introduce a dimensionless parameter, which relates the amount of accumulated vacancies at the $\eta$ '-Cu interface to the amount of the material diffused from this interface:

$$
P=\frac{\Phi^{\mathrm{vac}}}{\Phi^{\mathrm{Cu}}}
$$

This parameter is equal to zero when the total diffusion fluxes (including electromigration) of $\mathrm{Cu}$ and $\mathrm{Sn}$ are equal. In this case no vacancies will accumulate at this interface. Another limiting case when the total flux of $\mathrm{Cu}$ is much greater than the total flux of Sn (i.e., $P \rightarrow 1$ ) may result in $\mathrm{Cu}$ dissolution, see, e.g., [18].

Table 1

Model parameters used in computations [7, 17]

\begin{tabular}{|c|c|c|c|}
\hline Property & $\mathrm{Sn}$ & $\eta^{\prime}$ & $\mathrm{Cu}$ \\
\hline$E[\mathrm{GPa}]$ & 50 & 118 & 130 \\
\hline$v$ & 0.36 & 0.31 & 0.34 \\
\hline$\rho\left[\mathrm{g} / \mathrm{cm}^{3}\right]$ & 7.31 & 8.28 & 8.96 \\
\hline$\alpha[1 / \mathrm{K}]$ & $22 \cdot 10^{-6}$ & $18 \cdot 10^{-6}$ & $16.5 \cdot 10^{-6}$ \\
\hline$M[\mathrm{~g} / \mathrm{mol}]$ & 118.71 & 974.8 & 63.546 \\
\hline$\alpha_{*}[\mathrm{~cm} / \mathrm{s}]$ & $1 \cdot 10^{-3}$ & - & $2 \cdot 10^{-2}$ \\
\hline$k_{*}[\mathrm{~cm} / \mathrm{s}]$ & $1 \cdot 10^{-3}$ & - & $4 \cdot 10^{-2}$ \\
\hline$D\left[\mathrm{~cm}^{2} / \mathrm{s}\right]$ & $6.49 \cdot 10^{-12}$ & - & $7.04 \cdot 10^{-12}$ \\
\hline$Z^{*}$ & 36 & - & 26 \\
\hline$\varrho[\mathrm{Ohm} \cdot \mathrm{m}]$ & $1.15 \cdot 10^{-7}$ & - & $1.7 \cdot 10^{-8}$ \\
\hline
\end{tabular}

\section{Results}

The material parameters used for the calculations are compiled in Table 1 [7, 17]. Model and fitting parameters are chosen according to experimental results in [18]: $T=423[\mathrm{~K}], j=11\left[\mathrm{kA} / \mathrm{cm}^{2}\right], \alpha=15[\mu \mathrm{m}], b=18[\mu \mathrm{m}], \mathrm{c}=$ $150[\mu \mathrm{m}], A=0,8, n=1.642, \mathrm{Q}=0.8[\mathrm{eV}]$. The considered cases are listed in Table 2.

Table 2

Description of the analyzed cases

\begin{tabular}{|c|c|c|}
\hline Case number & Direction of $J^{\mathrm{EM}}$ and $J^{\mathrm{D}}$ & Effect of stresses \\
\hline 1 & co-directed & Yes \\
\hline 2 & directed opposite & Yes \\
\hline 3 & No EM & Yes \\
\hline $1^{\prime}$ & co-directed & No \\
\hline $2^{\prime}$ & directed opposite & No \\
\hline $3^{\prime}$ & No EM & No \\
\hline 4 & co-directed & Yes \\
\hline 5 & directed opposite & Yes \\
\hline 6 & No EM & Yes \\
\hline $4^{\prime}$ & co-directed & No \\
\hline $5^{\prime}$ & directed opposite & No \\
\hline $6^{\prime}$ & No EM & No \\
\hline
\end{tabular}

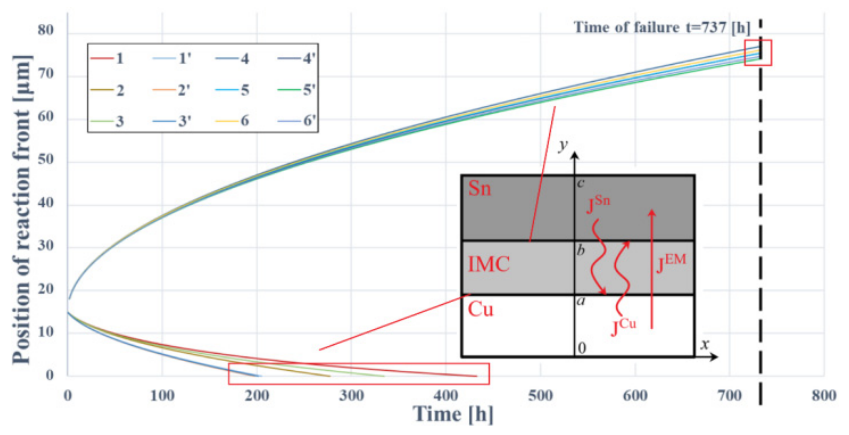

Fig. 3. Dependence of the position of the reaction front on time with and without taking stresses and electromigration into account. Labels in the legend of plot correspond to the case numbers in Table 2

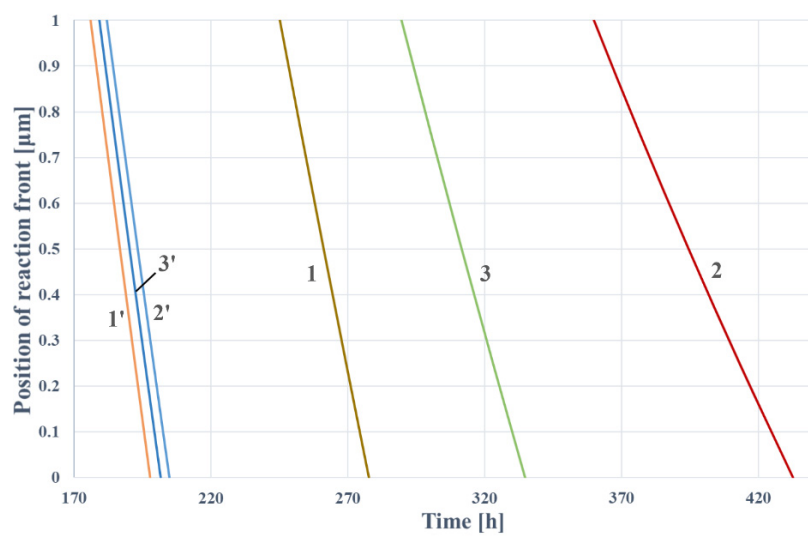

Fig. 4. Zoomed-in part of the reaction front position at $\eta^{\prime}-\mathrm{Sn}$ interface (bottom red square in Fig. 3). Curve numbers correspond to the case numbers in Table 2. 


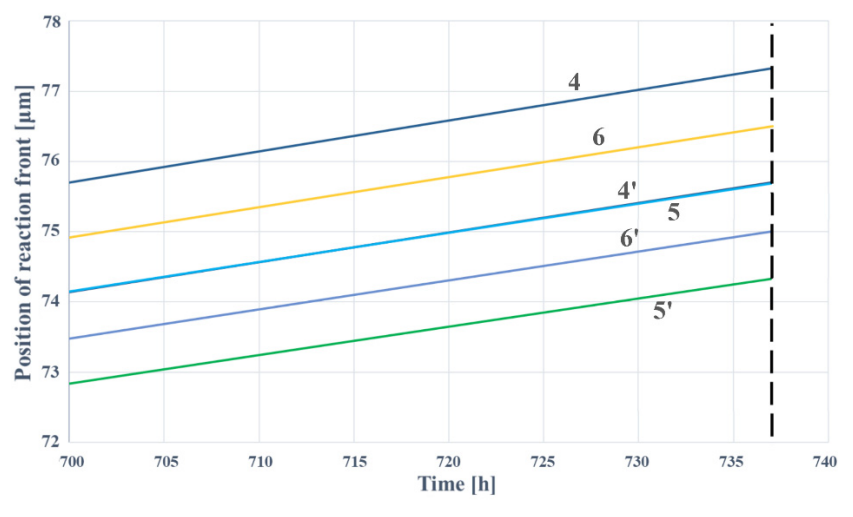

Fig. 5. Zoomed-in part of the reaction front position at $\eta^{\prime}-\mathrm{Cu}$ interface (upper red square in Fig. 3). Curve numbers correspond to the case numbers in Table 2

Fig. 3 presents the dependence of the positions of the reaction front on time for each of the considered cases. One can see two reactions happening simultaneously at different reaction fronts. The gap between initial positions of the reaction fronts is the initial thickness of IMC layer after soldering. It should be pointed out that electromigration accelerates the chemical reaction front if the flux induced by the electromigration is directed along with the Fickian flux and retards otherwise. It is also important to notice that the IMC-Cu interface propagates at least two times slower than the IMC-Sn interface. The detailed view in Fig. 5 shows that case 5 ( $J^{\mathrm{EM}}$ directed opposite to $J^{\mathrm{D}}$ ) and case 4 ' $\left(J^{\mathrm{EM}}\right.$ co-directed with $J^{\mathrm{D}}$ ) are indistinguishable. However, in case 5 the reaction front is retarded by the electromigration and accelerated by the stresses, and in case 4' the stresses are absent and the reaction front is accelerated by electromigration.

Two modes of failure can be observed in Fig. 3: full dissolution of $\mathrm{Cu}$ (lower set of curves); failure based on the MTTF criteria (upper set of curves reaching the vertical dashed line). The experimental study in [18] shows that the occurrence of the first failure mode ( $\mathrm{Cu}$ dissolution) is about $80 \%$ of and the second failure mode (MTTF criteria) occurs in only $20 \%$ of cases. In the current model we assume that if the first failure mode did not occur, yet all the $\mathrm{Cu}$ is dissolved, then the reaction interface $\eta^{\prime}-\mathrm{Cu}$ stops and remains stationary until the second failure mode occurs. According to (22) at the moment of failure the ratio of the accumulated vacancies to the amount of material diffused from $\eta^{\prime}-\mathrm{Cu}$ interface is 0.43 and 0.73 at the lower half and the upper half of the solder interconnect, respectively. Also, at the failure moment the total thickness of the IMC layer is $69.25 \mu \mathrm{m}$ and $69 \mu \mathrm{m}$ at the lower half and at the upper half of the solder, respectively. If the effects of stresses on the kinetics of the reaction front are neglected at the failure moment, the ratio of the accumulated vacancies to the amount of material diffused from $\eta^{\prime}-\mathrm{Cu}$ interface is 0.5 and 0.69 at the lower half and the upper half of the solder interconnect, respectively. Thus one can conclude that the presence of internal stresses affects the reliability of the solder interconnect negatively.

\section{Remark on voids nucleation}

In the previous section we considered vacancies kinetics and related fracture with some critical concentration of vacancies. Yet it is known that fracture may be preceded by void nucleation.

During the past decades several analytical and numerical models of void nucleation and growth were proposed. In work [28] it shown that if the grain boundary migration controls the diffusion process then vacancies rapidly accumulate at structural irregularities such as grain boundary triple point junction. The proposed model considered a spherical void nucleation at a triple point based on free energy change, and its further growth due to vacancy flux affected by thermal gradient and electromigration. Later, several studies were carried out to investigate the effect of mechanical stresses on the void nucleation: In [29] homogeneous nucleation of spherical voids under external and internal applied stresses was considered; in [30] the authors numerically modeled the influence of the plasticity; in [31] the authors used a stress dependent energy barrier for void nucleation and proposed an expression for the stress dependent diffusivity tensor of vacancies and investigated its effects on the void growth; in [32] the authors took the deformation produced by the vacancy migration and vacancy generation/annihilation and its influence on the void nucleation. It was shown that the presence of the mechanical stresses may result in the void nucleation without the local inhomogeneities [30, 31, 33]. Weinberg et al. [34-36] considered the formation and growth of the homogeneously distributed spherical voids. Also, various effects of void nucleation and growth were investigated: the influence of void formation on brittle/ductile fracture [37]; the increase of electrical resistance due to void formation [38]; the effect of temperature and tensile stresses on void nucleation/suppression [39]; the process of void nucleation and growth by means of nonequilibrium thermodynamics based on principle of maximum dissipation [40]. Furthermore, since the nucleated voids could move in the bulk and evolve their morphology due to electrical current, modeling the growth of Kirkendall voids gets increasingly complicated, therefore, numerical methods must be employed [41].

The change of the free energy due to void nucleation can be calculated as [42]:

$$
\Delta G=4 \pi r^{2} \gamma_{\text {surf }}-\frac{4}{3} \pi r^{3} \Delta f
$$

where $\gamma_{\text {surf }}$ is the surface tension, $r$ is the void radius, and $\Delta f$ is the change of the free energy due to void formation, which in the simplest case can be estimated as:

$$
\Delta f=\frac{k T}{V_{V}} \ln \left(\frac{c_{v}}{c_{v 0}}\right),
$$

where $V_{V}$ is the atomic volume, $c_{v}$ and $c_{v 0}$ are the vacancy concentration and equilibrium vacancy concentration, respectively. The latter is given by: 


$$
c_{v 0}=\exp \left(-\frac{\Delta f}{k T}\right) .
$$

Vacancies condense to form a void when the concentration is high enough to overcome the energy barrier. A relation between the concentration of vacancies and the radius of a void follows from (23) and (24):

$$
c_{v}=c_{v 0} \exp \left(\frac{2 \gamma_{\text {surf }} V_{V}}{k T r}\right) .
$$

In the model proposed in Section 3 it is assumed that the flux of vacancies is equal to the flux of atoms. Then, the concentration of the vacancies can be estimated as a difference between the concentration of the departed atoms of $\mathrm{Cu}$ and the $\mathrm{Sn}$ atoms that have arrived. Equating the right hand side of (26) to the concentration of vacancies estimated by (18), i.e., found from a solution of the mechanochemistry problem stated above, one can find a radius of the nucleated voids. Therefore, at more detailed consideration one has to take into account coupling of the chemical reaction kinetics, diffusion, electromigration, void nucleation and subsequent growth.

\section{Conclusion}

In this paper an analytical model of stress-affected kinetics of intermetallic compound $\mathrm{Cu}_{6} \mathrm{Sn}_{5}$ formation in the

\section{References}

1. S. Cheng, C.-M. Huang, and M. Pecht, A review of lead-free solders for electronics applications, Microelectronics Reliability 2017.

2. F. Mohd, C. Basaran, and Y.-S. Lai, Thermomigration versus electromigration in microelectronics solder joints, Advanced Packaging, IEEE Transactions 2009, Vol. 32, pp. 627-635.

3. B. Chao, S.-H. Chae, X. Zhang, K. Lu, M. Ding, J. Im, and P. Ho, Electromigration enhanced intermetallic growth and void formation in Pb-free solder joints, Journal of Applied Physics 2006, Vol. 100, 084909-084909.

4. A. Smigelskas and E. Kirkendall, Zinc diffusion in alpha brass, Trans. AIME 1947, Vol. 27, pp. 130-142.

5. B. Chao, X. Zhang, S.-H. Chae, and P. Ho, Recent advances on kinetic analysis of electromigration enhanced intermetallic growth and damage formation in pb-free solder joints, Microelectronics Reliability 2009, Vol. 49, pp. 253-263.

6. A. Paul, The Kirkendall effect in solid state diffusion, Ph.D. thesis, Department of Chemical Engineering and Chemistry 2004.

7. B. Chao, S.-H. Chae, X. Zhang, K. Lu, J. Im, and P. Ho, Investigation of diffusion and electromigration parameters for $\mathrm{Cu}-$ $\mathrm{Sn}$ intermetallic compounds in pb-free solders using simulated annealing, Acta Materialia 2007, Vol. 55, pp. 2805-2814.

8. G. Ross, V. Vuorinen, and M. Paulasto-Kröckel, Void formation and its impact on cu-sn intermetallic compound formation, Journal of Alloys and Compounds 2016, pp. 677.

9. T. Laurila, A. Paul, V. Vuorinen, and S. Divinski, Thermodynamics, diffusion and the Kirkendall effect in solids 2014, pp. 115-139, Cham: Springer International Publishing.

10. K. Zeng, R. Stierman, T.-C. Chiu, D. Edwards, K. Ano, and $\mathrm{K}$. N. Tu, Kirkendall void formation in eutectic $\mathrm{Sn}-\mathrm{Pb}$ solder joints on bare cu and its effect on joint reliability, Journal of Applied Physics 2005, Vol. 97. presence of the electromigration affected diffusion is presented. The kinetics of the chemical reaction fronts IMC$\mathrm{Cu}$ and IMC-Sn was investigated based on the notion of the chemical affinity tensor within the small strain approximation. It was shown that mechanical stresses produced by the chemical reaction and by the thermal strains result in the acceleration of the IMC-Cu reaction front with diffusing $\mathrm{Sn}$ and in the retardation of the IMC-Sn reaction front with diffusing $\mathrm{Cu}$. The flux induced by the electomigration results in the acceleration of the reaction front in the cases when it is co-directed with the Fickian flux and results in retardation if it is directed opposite to Fickian flux, as one could expect. A dimensionless parameter which estimates amount of accumulated vacancies at the IMC-Cu interface until the failure occurred was introduced and quantitatively estimated based on the MTTF criteria. In order to predict the reliability of the solder interconnects more accurately the current model may be extended to consider the effects of thermomigration, stress-migration, and polarity effects due to current crowding. Nucleation and growth of voids also requires further considerations.

\section{Acknowledgment}

The authors appreciate the support of the Russian Science Foundation (Grant No. 19-19-00552).

11. J. Kim, J. Yu, and S. Kim, Effects of sulfide-forming element additions on the Kirkendall void formation and drop impact reliability of cu/sn-3.5ag solder joints, Acta Materialia - ACTA MATER 2009, Vol. 57, pp. 5001-5012.

12. L. Xu, J. H. L. Pang, and F. Che, Impact of thermal cycling on sn-ag-cu solder joints and board-level drop reliability, Journal of Electronic Materials 2008, Vol. 37, pp. 880-886.

13. F. Gao and J. Qu, Calculating the diffusivity of $\mathrm{Cu}$ and $\mathrm{Sn}$ in $\mathrm{Cu}_{3} \mathrm{Sn}$ intermetallic by molecular dynamics simulations. Materials Letters 2012, Vol. 73, pp. 92-94.

14. I. A. Blech, Electromigration in thin aluminum films on titanium nitride, J. Appl. Phys. 1976, Vol.47, No.4, pp. 1203-1208.

15. A. B. Freidin and E. N. Vilchevskaya, Chemical affinity tensor in coupled problems of mechanochemistry, in Encyclopedia of Continuum Mechanics, edited by H. Altenbach and A. Öchsner (Springer Berlin Heidelberg, Berlin, Heidelberg, 2019) pp. 1-17.

16. A. Freidin, E. Vilchevskaya, and I. Korolev, Stress-assist chemical reactions front propagation in deformable solids, International Journal of Engineering Science 2014, Vol. 83, pp. 57-75.

17. A. Morozov, S. Khakalo, V. Balobanov, A. Freidin, W. H. Müller, and J. Niiranen, Modeling chemical reaction front propagation by using an isogeometric analysis, Technische Mechanik 2018, Vol. 38, pp. 73-90.

18. J.-Y. Park, T. Lee, W. Seo, S. Yoo, and Y.-H. Kim, Electromigration reliability of $\mathrm{Sn}-3.0 \mathrm{Ag}-0.5 \mathrm{Cu} / \mathrm{Cu}-\mathrm{Zn}$ solder joints, Journal of Materials Science: Materials in Electronics 2019, Vol. 30, pp. 7645-7653.

19. M. Poluektov, A. Freidin, and Ł. Figiel, Modelling stressaffected chemical reactions in non-linear viscoelastic solids with application to lithiation reaction in spherical Si particles, International Journal of Engineering Science 2018, Vol. 128, pp. 44-62. 
20. A. Morozov, A. B. Freidin, V. A. Klinkov, A. V. Semencha, W. H. Müller, and T. Hauck, Experimental and theoretical studies of $\mathrm{Cu}-\mathrm{Sn}$ intermetallic phase growth during hightemperature storage of eutectic snag interconnects, Journal of Electronic Materials 2020.

21. A. Morozov, A. Freidin, W. H. Müller, A. Semencha, and M. Tribunskiy, Modeling temperature dependent chemical reaction of intermetallic compound growth, in 2019 20th International Conference on Thermal, Mechanical and Multi-Physics Simulation and Experiments in Microelectronics and Microsystems (EuroSimE 2019) pp. 1-8.

22. P. Glansdorff, I. Prigogine, and R. N. Hill, Thermodynamic theory of structure, stability and fluctuations, American Journal of Physics 1973, Vol. 41, pp. 147-148.

23. A. B. Freidin, Chemical affinity tensor and stress-assist chemical reactions front propagation in solids, in Proceedings of the ASME 2013 International Mechanical Engineering Congress and Exposition, Vol. 9 (American Society of Mechanical Engineers, 2013) p. V009T10A102

24. K. N. Tu, Fundamentals of electromigration, in Springer Series in Materials Science, Springer Series in Materials Science, Springer Verlag 2007, pp. 211-243.

25. C. Chen, H. Tong, and K. Tu, Electromigration and thermomigration in $\mathrm{Pb}$-free flip-chip solder joints, Annual Review of Materials Research 2010, Vol. 40, pp. 531-555.

26. A. G. Knyazeva, Cross effects in solid media with diffusion, Journal of Applied Mechanics and Technical Physics 2003, Vol. 44.3, pp. 373-384.

27. J. R. Black, Electromigration - a brief survey and some recent results, IEEE Transactions on Electron Devices 1969, Vol. 16, pp. 338-347.

28. R. Rosenberg and M. Ohring, Void formation and growth during electromigration in thin films. Journal of Applied Physics 1971, Vol. 42(13), pp. 5671-5679.

29. J. P. Hirth and W. D. Nix, Analysis of cavity nucleation in solids subjected to external and internal stresses. Acta Metallurgica 1985, Vol. 33(3), pp. 359-368.

30. C. Basaran and M. Lin, Damage mechanics of electromigration in microelectronics copper interconnects, Intl. J. Materials and Structural Integrity 2007, Vol. 1, pp. 16-39.
31. H. Ceric, R. L. de Orio, J. Cervenka, and S. Selberherr, A comprehensive TCAD approach for assessing electromigration reliability of modern interconnects, IEEE Trans. Mat. Dev. Rel. 2009, Vol. 9, No. 1, pp. 9-19.

32. R. V. Goldstein, M. E. Sarychev, D. B. Shirabaikin, A. S. Vladimirov, Y. V. Zhitnikov, Modeling of electromigration and the void nucleation in thin-film interconnects of integrated circuits. International Journal of Fracture. 2001, Vol. 109, pp. 91-121.

33. R. Kirchheim, Stress and electromigration in Al-lines of integrated circuits, Acta Metall. Mater. 1992, Vol. 40, No. 2, pp. 309-323.

34. K. Weinberg, T. Böhme, and W. H. Müller, Kirkendall voids in the intermetallic layers of solder joints in MEMS. Computational Materials Science 2009, Vol. 45(3), pp. 827-831

35. K. Weinberg and T. Böhme, Condensation and growth of Kirkendall voids in intermetallic compounds. IEEE Transactions on Components and Packaging Technologies 2009, Vol. 32(3), pp. $684-692$

36. K. Weinberg, T., Böhme, Mesoscopic modeling for continua with pores. Dynamic void growth in viscoplastic materials. Journal of Non-Equilibrium Thermodynamics 2008, Vol. 33(1), pp. 25-45.

37. A. M. Cuitiño, and M. Ortiz, Ductile fracture by vacancy condensation in f.c.c. single crystals. Acta Materialia 1996, Vol. 44(2), pp. 427-436.

38. R. Kirchheim and U. Kaeber, Atomistic and computer modeling of metallization failure of integrated circuits by electromigration. Journal of Applied Physics 1991, Vol. 70(1), pp. 172-181.

39. V.I. Levitas and H. Attariani, Mechanochemical continuum modeling of nanovoid nucleation and growth in reacting nanoparticles. The Journal of Physical Chemistry 2011, Vol. 116(1), pp. 54-62.

40. Fischer, F. D., Svoboda, J. Void growth due to vacancy supersaturation - A non-equilibrium thermodynamics study, Scripta Materialia 2008, Vol. 58(2), pp. 93-95

41. H. Ceric and S. Selberherr, Electromigration modeling for interconnect structures in microelectronics, ECS Transactions 2007, Vol. 9 (1), pp. 295-304.

42. Y.E. Gegusin. Diffusion Zone, Nauka, Moscow, (1979), in Russian. 